\title{
Short-course Versus Long-course Preoperative Radiotherapy plus Delayed Surgery in the Treatment of Rectal Cancer: a Meta-analysis
}

\author{
Shi-Xin Liu ${ }^{1 \& *}$, Zhi-Rui Zhou ${ }^{2 \&}$, Ling-Xiao Chen ${ }^{3}$, Yong-Jing Yang ${ }^{1}$, Zhi-De Hu ${ }^{4}$, \\ Tian-Song Zhang ${ }^{5}$
}

\begin{abstract}
Background: Short-course preoperative radiation (SCRT) with delayed surgery was found to increase pathologic complete response (pCR) rates in several trials. However, there was no clear answer on whether SCRT or long-course chemo-radiotherapy (LCRT) is more effective. Therefore we conducted this meta-analysis to evaluate the safety and efficacy of SCRT versus LCRT, both with delayed surgery, for treatment of rectal cancer. Materials and Methods: The literature was searched from PubMed, EMBASE, Web of Science, Cochrane Library and clinicaltrials.gov up to November, 2014. Quality of the randomized controlled trials (RCTs) was evaluated according to the Cochrane's risk of bias tool of RCT. The Grading of Recommendations Assessment, Development and Evaluation (GRADE) system was used to rate the level of evidence. Review Manager 5.3 was employed for statistical analysis. Pooled risk ratios (RRs) and 95\% confidence intervals (CIs) were calculated. Results: Three RCTs, with a total of 357 rectal cancer patients, were included in this systematic review. Metaanalysis results demonstrated there were no significantly differences in sphincter preservation rate, local recurrence rate, grade $3 \sim 4$ acute toxicity, $R 0$ resection rate and downstaging rate. Compared with SCRT, LCRT was associated with significant increase in the $\mathrm{pCR}$ rate $[R R=0.49,95 \% \mathrm{CI}(0.31,0.78), P=0.003]$. Conclusions: In terms of sphincter preservation rate, local recurrence rate, grade $3 \sim 4$ acute toxicity, $\mathrm{R} 0$ resection rate and downstaging rate, SCRT with delayed surgery is as effective as LCRT with delayed surgery for management of rectal cancer. LCRT significantly increased PCR rate compared with SCRT. Due to risk of bias and imprecision, further multi-center large sample RCTs were needed to confirm this conclusion.
\end{abstract}

Keywords: Rectal cancer - preoperative radiotherapy - chemo-radiotherapy - meta-analysis.

Asian Pac J Cancer Prev, 16 (14), 5755-5762

\section{Introduction}

Long-course chemo-radiotherapy (45 50 Gy in 25 fractions) with delayed surgery or short-course radiotherapy ( 25 Gy in 5 fractions) with immediate surgery were the most frequent regimens for the treatment of localized and locally advanced resectable rectal cancer (SWEDISH RECTAL CANCER TRIAL, 1997; Colorectal Cancer Collaborative Group, 2001; Kapiteijn et al., 2001; Sauer et al., 2004). Compared with postoperative chemo-radiotherapy, this neoadjuvant strategy improved local control and was associated with reduced toxicity and better compliance of radiotherapy (Frykholm et al., 1993; Sauer et al., 2004; Sebag-Montefiore et al., 2009). The MRC (Medical Research Council) CR07 rectal trial further supported for the short course preoperative regimen (Sebag-Montefiore et al., 2009). Long-course preoperative chemo-radiotherapy (LCRT) of 50.4 Gy in about 6 weeks with delayed surgery has been widely used in the last decades, which superiority, in terms of local control, was demonstrated in the German rectal cancer trial, compared with postoperative chemo-radiotherapy (Sauer et al., 2004; Sauer et al., 2012).

To date, many studies have explore whether SCRT or LCRT is more effective neoadjuvant modality to improve outcomes for localized and locally advanced rectal cancer, but the results were heterogeneous (Bujko and Bujko, 2011; Minsky, 2011; Minsky et al., 2014; Palta et al., 2014). On the one hand, the benefit of the short-course schedule is less expensive and more convenient, especially

${ }^{1}$ Department of Radiation Oncology, Tumor Hospital of Jilin Province, Changchun, ${ }^{2}$ Department of Radiation Oncology, Fudan University Shanghai Cancer Center; Department of Oncology, Shanghai Medical College, Fudan University, ${ }^{5}$ Internal Medicine of Traditional Chinese Medicine Department, Jing 'an District Central Hospital of Shanghai, Shanghai, ${ }^{3}$ Department of Orthopaedics, Tianjin Medical University General Hospital, Tianjin, ${ }^{4}$ Department of Laboratory Medicine, General Hospital of Ji'nan Military Command Region, Ji'nan, China \&Equal contributors *For correspondence: liushixin1964@126.com 
in centers with a long waiting list or lack of medical resources (Bujko et al., 2006; Zhou et al., 2014). On the other hand, long-course chemo-radiation might be better than the short-course irradiation schedule at increasing pathologic complete response (pCR) and R0 resection rate (Bujko et al., 2006; Zhou et al., 2014), because the tumor bulk might be reduced before surgery.

Ameta-analysis was conducted by our team manifested that SCRT with immediate surgery is as effective as LCRT with delayed surgery for treatment of rectal cancer in terms of survival, recurrence, sphincter preservation rate, $\mathrm{R} 0$ resection rate and late toxicity. Though LCRT increased pCR rate, LCRT also increased acute toxicity compared with SCRT (Zhou et al., 2014). In fact, the optimal timing interval of surgery remains controversial. Short-course preoperative radiation with delayed surgery has been shown to increase pathologic complete response (pCR) rate and induce tumour down-staging rate in both randomized and observational studies in recent years (Radu et al., 2008; Hatfield et al., 2009; Pettersson et al., 2010). Particularly worth mentioning is that preoperative radiotherapy for rectal carcinoma is worrying in developing Asia country, because this regimen was with the high rate of local recurrence (Lee et al., 2013). To facilitate clinical decision making, summarizing the available evidence is particularly urgent. Therefore, we performed this meta-analysis to fully evaluate the safety and efficacy of SCRT with delayed surgery versus LCRT with delayed surgery as a modality for the management of rectal cancer.

\section{Materials and Methods}

\section{Inclusion criteria}

Studies would be included in systematic review if they met the following criteria: (i) All patients that were diagnosed as localized and locally advanced resectable rectal cancer using pathology and cytology were included in systematic review. Metastatic rectal cancer patients were excluded. All the patients did not have serious cardiopulmonary diseases and other severe underlying diseases. (ii) Comparing the efficacy and safety of shortcourse radiotherapy with delayed surgery +/- adjuvant chemotherapy versus long-course radiotherapy or chemo-radiotherapy with delayed surgery +/- adjuvant chemotherapy for treatment of rectal cancer. Short-course radiotherapy was not more than one week, and long-course radiotherapy should be more than 4 weeks at least. Total dose of short-course was at least more than $20 \mathrm{~Gy}$, and long-course surpassed $45 \mathrm{~Gy}$. Timing interval of surgery was more than 4 weeks either SCRT or LCRT arms. (iii) The following outcomes were evaluated: sphincter preservation rate, $\mathrm{R} 0$ resection rate, Downstaging ( $\mathrm{T}$ stage decreased) rate, $\mathrm{pCR}$ rate, local recurrence rate and grade 3 4 acute toxicity. (iv) Randomized control trials (RCTs).

We excluded the following articles: (i) The design of the study was not randomized controlled trials, for example, retrospective study, case series or case report etc.; (ii) The study had repeated data or did not report outcomes of interest; (iii) Non-original research, such as review, comment and letter etc.; (iv) Short-course radiotherapy was more than one week, or long-course radiotherapy less than 4 weeks. Total dose of short-course was less than $20 \mathrm{~Gy}$, or long-course less than $45 \mathrm{~Gy}$. Timing interval of surgery was less than 4 weeks either SCRT or LCRT arms.

Eligibility assessment was performed independently in a non-blinded standardized manner by 2 reviewers. Any disagreement between two authors was resolved by discussion.

\section{Literature search}

We searched related articles in PubMed (From 1966 to November 2014), EMBASE (From 1974 to November 2014), Web of Science, Cochrane Library (CENTRAL, Issue 11 of 12, November 2014) and clinicaltrials.gov up to November, 2014. In electronic searching, we used MeSH or Emtree terms combined free terms in all the search strategies. The following search terms were used: "rectal cancer", "preoperative radiotherapy", "chemoradiotherapy", "neoadjuvant radiotherapy", "short course" and "long course". The whole search strategies were listed in the appendix. We also reviewed the references of included studies to look for potentially eligible articles. Furthermore, we checked abstracts that were published in major academic conferences (American Society of Clinical Oncology, European Society for Medical Oncology, American Society for Therapeutic Radiology and Oncology and European SocieTy for Radiotherapy \& Oncology). No language restrictions were adopted.

\section{Assessing risk of bias of included RCTs}

Qualities of included RCTs were evaluated according to the Cochrane Collaboration's tool for assessing risk of bias of RCT (ROB tool, 5.1 .0) (Higgins and Green, 2011). The RoB tool included seven domains: sequence generation, allocation concealment, blinding of participants, personnel and outcome assessors, incomplete outcome data, selective outcome reporting, and other sources of bias. For each study, we made judgments about risk of bias from each of the seven domains of the tool. In all domains, an answer 'Yes' indicated a low risk of bias, an answer ' $\mathrm{No}$ ' indicated high risk of bias, and if insufficient detail is reported of what happened in the study, the judgment would usually be 'Unclear' risk of bias.

\section{Data extraction}

Data extraction was performed completely independently by two reviewers. Reviewers were not blinded to authors or journals. Disagreements were resolved by discussion between the two review authors; if no consensus was reached, a third author would decide. Information about baseline patient characteristics, duration of follow up, and the number of events for all the outcomes, trial design, interventions and outcomes were extracted from each included study.

\section{Data analysis}

All statistical analyses were performed using Review Manager 5.3 software. Risk ratio (RR) and 95\% confidence intervals (CI) was calculated for count data. 
Chi-square test and I-square test were used for testing heterogeneity between studies. If heterogeneity was not present $\left(P>0.10, \mathrm{I}^{2}<50 \%\right)$, fixed-effect model would be adopted for analysis, otherwise, random-effect would be employed. In the presence of heterogeneity, we explored potential sources from the following three aspects: clinical, methodological and statistical. In the case of excessive heterogeneity, descriptive analysis rather than metaanalysis was employed.

\section{Quality of evidence}

The Grading of Recommendations Assessment, Development, and Evaluation (GRADE) approach was used to grade the quality of evidence and strength of recommendations (Balshem et al., 2011; Guyatt et al., 2011a; Guyatt et al., 2011b; Guyatt et al., 2011c). Risk of bias, limitations, the indirectness, the consistency of the results across studies, the precision of the overall estimate across studies and other considerations are six domains of the tool. For each outcome, if further research was very unlikely to change our confidence in the estimate of effect, the quality of the evidence was rated as high; if further research was likely to have an important impact on our confidence in the estimate of effect and may change the estimate, the quality was moderate; if further research was very likely to have an important impact on our confidence in the estimate of effect and is likely to change the estimate, the quality was low; if the estimate was very uncertain, the quality was very low. The GRADEpro 3.6 software was used to estimate the quality of the evidence in the meta-analysis by two reviewers. If there were disagreements between the two reviewers, a third author would join to discuss and make decisions.

\section{Results}

Study selection and characteristics of included studies

Total 502 relevant literatures were searched, 165 duplicates were removed. After reviewed the titles and abstracts of 337 of records, 330 of them was excluded due to significant irrelevancy. 7 of full-text were obtained to further determine eligibility. We ruled out another 3 of full-text articles: 1 article due to interventions not meeting inclusion criteria; 1 article due to review; 1 article due to non-RCT. Finally, 4 studies were included in the qualitative systematic review (Pettersson et al., 2010; Latkauskas et al., 2012; Bujko et al., 2013; Nilsson et al., 2013), 1 of them is an ongoing trial, no publications were reported (Nilsson et al., 2013); 3 trials, total 357 patients, were included in meta-analysis (Pettersson et al., 2010; Latkauskas et al., 2012; Bujko et al., 2013). The PRISMA flow diagram of studies was shown in Figure 1. The characteristics of the studies were shown in Table 1.

\section{Risk of bias assessment}

This meta-analysis included 3 RCTs: the baseline characteristics of patients were reported in all RCTs. All 3 RCTs mentioned "random"; only 1 RCT reported an adequate randomized sequence generation and allocation concealment (Pettersson et al., 2010), 1 RCT was unclear (Latkauskas et al., 2012), 1 RCT was with high risk (Bujko et al., 2013). 2 RCTs described the reasons of incomplete outcome data (Pettersson et al., 2010; Latkauskas et al., 2012), 1 RCT with high risk (Bujko et al., 2013). All trials did not mention whether the blind method was adopted or not, however, this should unlikely affect the quality assessment results (Figure 2).

\section{Results of meta-analysis}

Sphincter preservation rate: Two trials reported the sphincter preservation rate; total 172 rectal cancer patients were included in the meta-analysis. There was no significant difference in the sphincter preservation rate between SCRT and LCRT arms $[\mathrm{RR}=1.14,95 \% \mathrm{CI}$ $(0.86,1.52), p=0.37]$. No obvious heterogeneity was found $\left(\mathrm{I}^{2}=24 \%, P=0.25\right)$, so the fixed effect model was employed, (Figure 3A).

\section{$R 0$ resection rate}

Two trials, with total 127 rectal cancer patients, were included in the meta-analysis to evaluate R0 resection

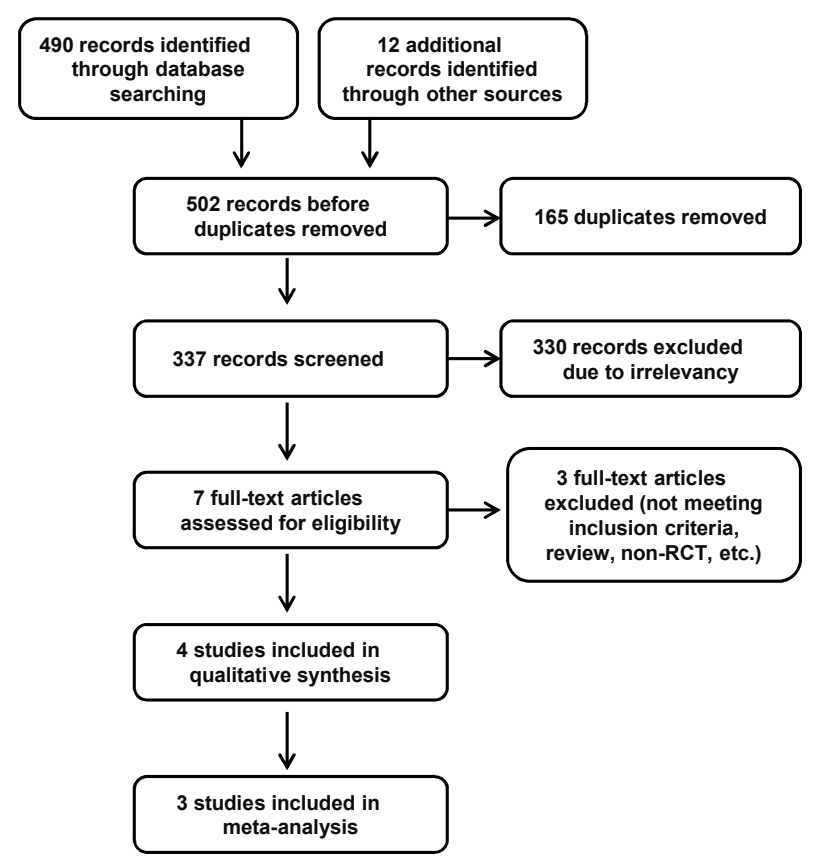

Figure 1. Flow Chart of the Study Selection Process

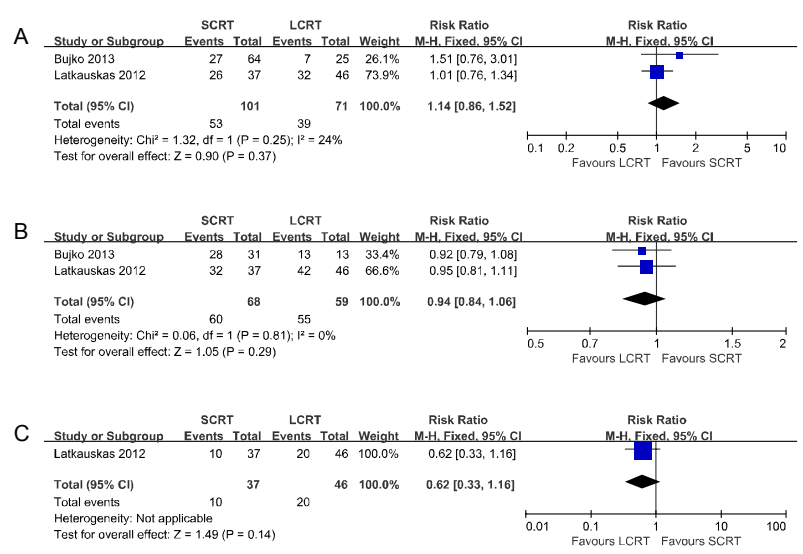

Figure 3. Meta-analysis of Short-course RT plus delayed surgery $v$ s. long-course chemo-RT plus delayed surgery. (A): Meta-analysis results of Sphincter preservation rate; (B): Meta-analysis results of R0 resection rate; (C): Metaanalysis results of downstaging rate 
rate. Meta-analysis suggested that there was no significant difference between SCRT and LCRT arms [RR=0.94, 95\% CI $(0.84,1.06), p=0.29]$, without significant heterogeneity was detected $\left(\mathrm{I}^{2}=0 \%, P=0.81\right)$, so the fixed effect model was applicable (Figure 3B).

\section{Downstaging (T stage decreased) rate}

Only one RCT evaluate the downstaging rate. Compared SCRT with LCRT, there was no significant difference between the two arms $[\mathrm{RR}=0.62,95 \% \mathrm{CI}(0.33$, 1.16), $P=0.14$ ] (Figure 3C).

\section{$p C R$ rate}

Two RCTs reported the pCR rate; total 172 rectal cancer patients were included in the meta-analysis. LCRT significantly increased the pCR rate compared with SCRT [RR $=0.49,95 \% \mathrm{CI}(0.31,0.78), P=0.003]$, without heterogeneity was found $\left(\mathrm{I}^{2}=0 \%, p=0.32\right)$, so fixed effect model was adopted (Figure 4A).

\section{Local recurrence rate ( $L R R)$}

Only one RCT reported the local recurrence rate. There was no significant difference between SCRT and LCRT arms [RR=4.76, 95\%CI $(0.66,34.53), p=0.12]$, (Figure 4B).

\section{Grade 3 4 acute toxicity}

Two RCTs reported the grade 3 4 acute toxicity; total 274 rectal cancer patients were included in the metaanalysis. There was no significant difference between

Table 1. Characteristics of Trials Included in Systematic Review

\begin{tabular}{|c|c|c|c|c|c|c|c|c|}
\hline Study & Arms & $\begin{array}{l}\text { Sample } \\
\text { Size }\end{array}$ & $\begin{array}{l}\text { Inclusion } \\
\text { Periods }\end{array}$ & $\begin{array}{c}\text { Follow- } \\
\text { up } \\
\text { Time }\end{array}$ & $\begin{array}{l}\text { Design } \\
\text { of } \\
\text { Study }\end{array}$ & Stage & Interventions & Outcomes \\
\hline \multirow{3}{*}{$\begin{array}{l}\text { Pettersson } \\
\text { et al } 2010\end{array}$} & SCRT & 118 & 1998 to & l & RCT & Stage & $\begin{array}{l}\text { 5x5Gy RT and TME surgery } \\
\text { within } 1 \text { week; }\end{array}$ & Acute toxicity \\
\hline & $\begin{array}{l}\text { SCRT- } \\
\text { delay }\end{array}$ & 120 & 2005 & & & $\mathrm{I} \sim \mathrm{IV}$ & $\begin{array}{l}\text { 5x5Gy RT and TME surgery } \\
\text { after } 4 \sim 8 \text { weeks; }\end{array}$ & \\
\hline & $\begin{array}{l}\text { LCRT- } \\
\text { delay }\end{array}$ & 65 & & & & & $\begin{array}{c}25 \times 2 G y \text { and TME after } 4 \sim 8 \\
\text { weeks later }\end{array}$ & \\
\hline \multirow{4}{*}{$\begin{array}{l}\text { Latkauskas } \\
\text { et al } 2012\end{array}$} & $\begin{array}{l}\text { SCRT- } \\
\text { delay }\end{array}$ & 37 & 2007 to & l & RCT & Clinical & $\begin{array}{c}25 \mathrm{~Gy} \text { in } 5 \text { fractions over } 5 \\
\text { days }\end{array}$ & $\begin{array}{l}\mathrm{R} 0 \text { resection } \\
\text { rate }\end{array}$ \\
\hline & $\begin{array}{l}\text { LCCRT- } \\
\text { delay }\end{array}$ & 46 & 2010 & & & stage & $\begin{array}{c}\text { followed surgery after } 6 \\
\text { weeks; }\end{array}$ & $\begin{array}{c}\text { Sphincter } \\
\text { preservation } \\
\text { rate }\end{array}$ \\
\hline & & & & & & II III & $\begin{array}{c}\text { 50Gy in } 25 \text { fractions over } 5 \\
\text { weeks with }\end{array}$ & $\begin{array}{l}\text { Postoperative } \\
\text { complication }\end{array}$ \\
\hline & & & & & & & $\begin{array}{l}5-\mathrm{Fu} / \mathrm{Lv} \text { followed surgery } \\
\text { after } 6 \text { weeks }\end{array}$ & $\begin{array}{l}\text { Pathological } \\
\text { downstaging }\end{array}$ \\
\hline \multirow{5}{*}{$\begin{array}{l}\text { Bujko et al } \\
2013\end{array}$} & $\begin{array}{l}\text { SCRT- } \\
\text { delay }\end{array}$ & 64 & 2003 to & 24 & RCT & Unfavorable & $\begin{array}{c}25 \mathrm{~Gy} \text { in } 5 \text { fractions over one } \\
\text { week }+4 \mathrm{~Gy} \text { boost }\end{array}$ & $\begin{array}{l}\text { Local } \\
\text { recurrence } \\
\text { rate }\end{array}$ \\
\hline & $\begin{array}{l}\text { LCRT- } \\
\text { delay }\end{array}$ & 25 & 2010 & $(2 \sim 85)$ & & $\mathrm{cT} 1 \sim 3 \mathrm{~N} 0$ & $\begin{array}{l}\text { Local excision was } \\
\text { performed } 6 \sim 8 \text { weeks later; }\end{array}$ & $\begin{array}{l}\text { Acute } \\
\text { radiation } \\
\text { toxicity }\end{array}$ \\
\hline & & & & & & & $\begin{array}{c}50.4 \mathrm{~Gy} \text { in } 28 \text { fractions plus } \\
5.4 \mathrm{~Gy} \text { boost }\end{array}$ & pCR rate \\
\hline & & & & & & & $\begin{array}{c}\text { concurrent } 3 \text { cycles of } \\
5-\mathrm{FU} / \mathrm{Lv} \text {, Local }\end{array}$ & \\
\hline & & & & & & & $\begin{array}{c}\text { excision was performed } 6 \sim 8 \\
\text { weeks later }\end{array}$ & \\
\hline \multirow{3}{*}{$\begin{array}{l}\text { Nilsson et } \\
\text { al } 2013\end{array}$} & $\begin{array}{l}\text { SCRT- } \\
\text { delay }\end{array}$ & I & I & l & RCT & cT4N2 & $\begin{array}{l}5 \mathrm{Gyx} 5 \text { followed } \\
\text { capecitabine and }\end{array}$ & $\begin{array}{c}\text { DFS, OS, } \\
\text { Toxicity }\end{array}$ \\
\hline & $\begin{array}{c}\text { LCCRT- } \\
\text { delay }\end{array}$ & I & & & & & $\begin{array}{c}\text { oxaliplatin in } 6 \text { cycles } \\
\text { before TME; }\end{array}$ & $\begin{array}{l}\mathrm{R} 0 \text { rate, } \mathrm{pCR} \\
\text { rate }\end{array}$ \\
\hline & & & & & & & $\begin{array}{c}1.8 \mathrm{Gyx} 25 \text { or } 2 \mathrm{Gyx} 25 \text { with } \\
\text { capecitabine followed }\end{array}$ & Quality of life \\
\hline ongoing & & & & & & & $\begin{array}{c}\text { TME plus selective } \\
\text { postoperative adjuvant } \mathrm{CT}\end{array}$ & \\
\hline
\end{tabular}

*Abbreviations: SCRT=short-course radiotherapy, LCRT=long-course radiotherapy, SCCRT=short course chemo-radiotherapy, LCCRT=longcourse chemo-radiotherapy, $\mathrm{CT}=$ chemotherapy, $\mathrm{RT}=$ radiotherapy, $\mathrm{TME}=$ total mesorectal excision, $\mathrm{OS}=$ overall survival, $\mathrm{DFS}=$ disease free survival, $\mathrm{pCR}=$ pathologic complete response, $\mathrm{Lv}=$ leucovorin 


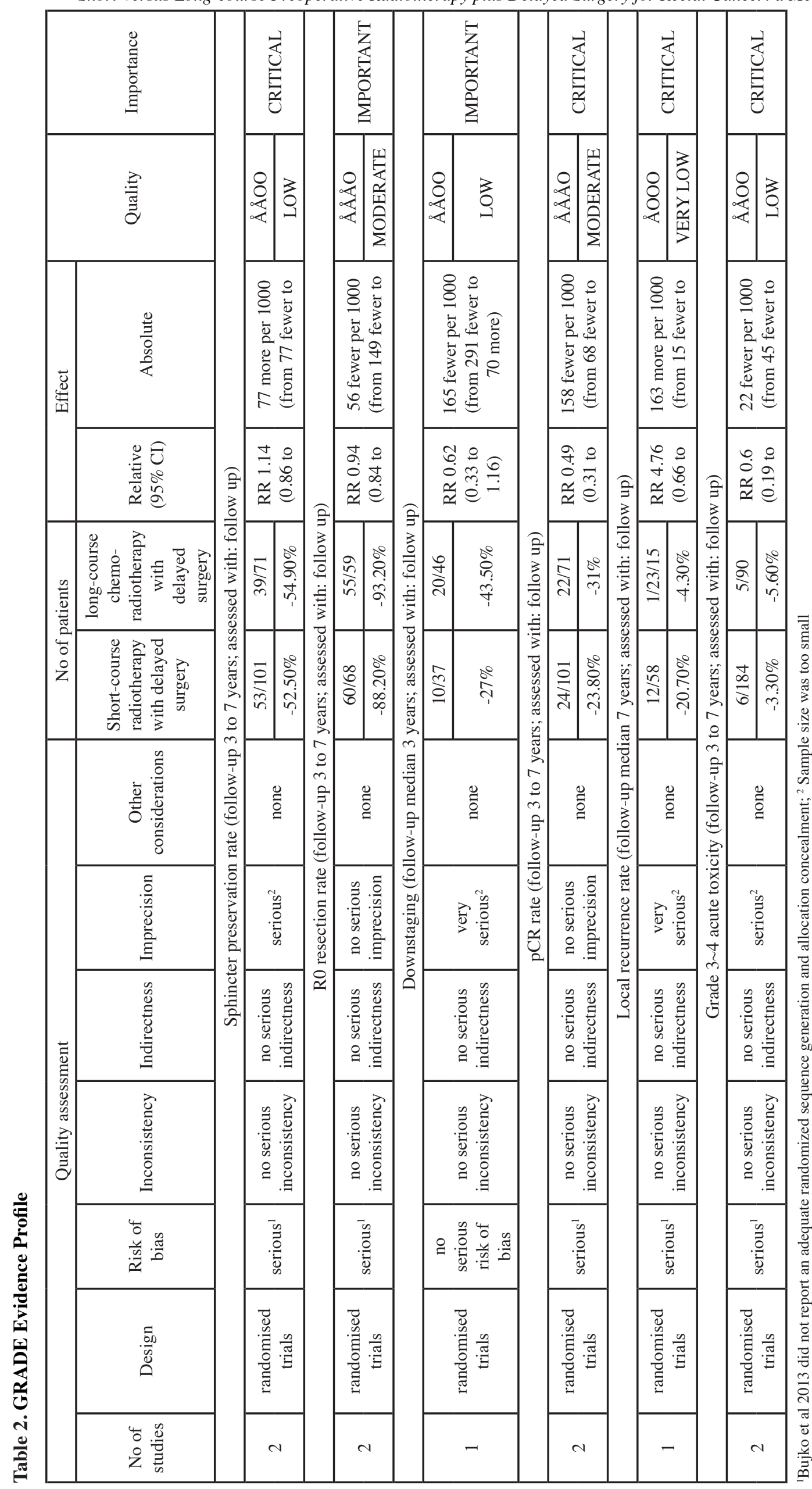




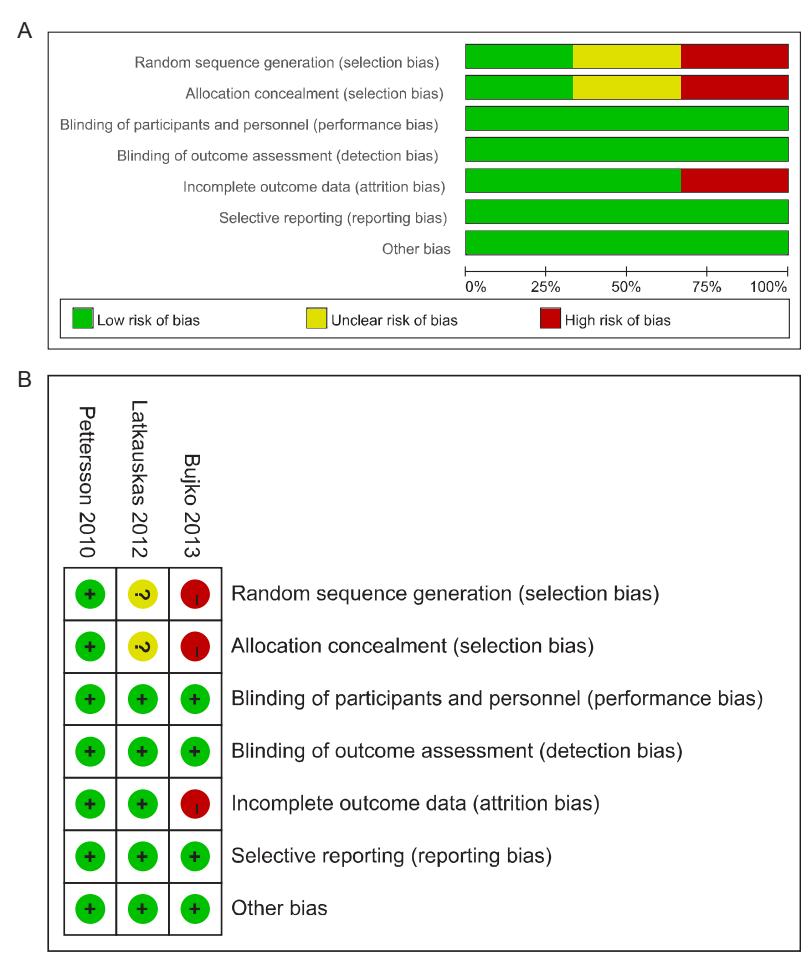

Figure 2. A) Risk of bias graph: review authors' judgments about each risk of bias item presented as percentages across all included studies; (B) Risk of bias summary: review authors' judgments about each risk of bias item for each included study

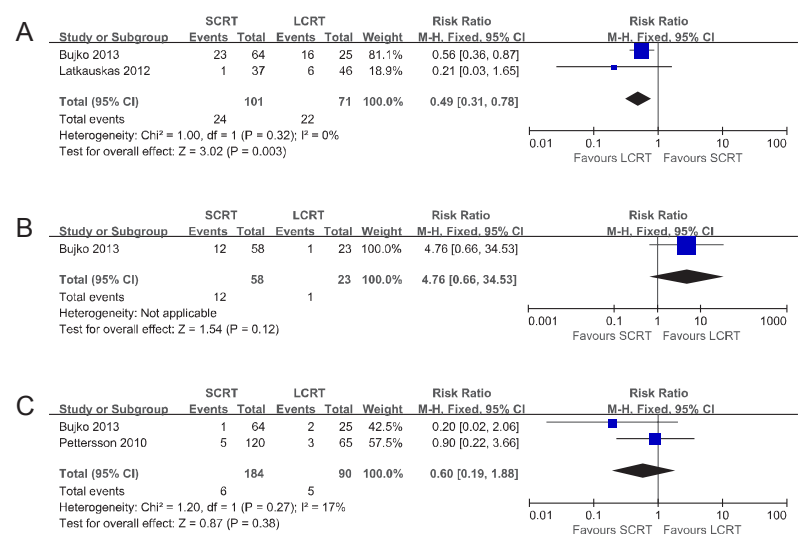

Figure 4. Meta-analysis of Short-course RT Plus Delayed Surgery vs. Long-course Chemo-RT Plus delayed surgery. (A): Meta-analysis results of pCR rate; (B): Meta-analysis results of Local recurrence rate (LRR); (C): Meta-analysis results of Grade 3 4 acute toxicity

SCRT and LCRT arms [RR $=0.60,95 \% \mathrm{CI}(0.19,1.88)$, $P=0.38]$, without obvious heterogeneity was detected $\left(\mathrm{I}^{2}=17 \%, p=0.27\right)$, so the fixed effect model was employed, (Figure 4C).

\section{Quality of evidence}

There were 6 outcomes in this meta-analysis. Sphincter preservation rate, $\mathrm{pCR}$ rate, local recurrence rate and grade $3 \sim 4$ acute toxicity were critical outcomes; R0 resection rate and downstaging rate were both important outcomes. The quality of the evidence of each outcome was shown in Table 2.

\section{Discussion}

Main findings: This systematic review and metaanalysis manifested that there were no significant differences in sphincter preservation rate, local recurrence rate, grade 3 4 acute toxicity, $\mathrm{R} 0$ resection rate and downstaging rate between two arms. Compared with SCRT plus delayed surgery, LCRT with delayed surgery significant increased $\mathrm{pCR}$ rate $[\mathrm{RR}=0.49,95 \% \mathrm{CI}(0.31$, $0.78), P=0.003]$. Based on the GRADE system, the evidence qualities of $\mathrm{pCR}$ rate and $\mathrm{R} 0$ resection rate were "moderate"; the evidence qualities of sphincter preservation rate, downstaging rate and grade $3 \sim 4$ acute toxicity were "low"; local recurrence rate was "very low". The main reason was risks of bias and imprecision.

Agreements and disagreements in the current literature: As far as we know, this is the first meta-analysis to compare short-course with long-course preoperative radiotherapy plus delayed surgery in the treatment of rectal cancer. Previous reviews mainly focused on SCRT plus immediate surgery, surgery alone, LCRT plus delayed surgery (Kye and Cho, 2014) or SCRT plus immediate surgery versus LCRT plus delayed surgery (Bujko et al., 2014; Zhou et al., 2014); They indicated both short-course plus immediate surgery and long-course preoperative radiotherapy plus delayed surgery were efficient and safety methods to treat rectal cancer. Further, our article direct compared short-course and long-course preoperative radiotherapy plus delayed surgery, the synthetic results are mostly consist with results from included studies in view of risk ratios and $95 \% \mathrm{CI}$.

Overall completeness and applicability of evidence: Most of rectal cancer patients included in this metaanalysis were clinical stage II III and without distant metastasis. Thus the results of this meta-analysis could be applicable to the patients with localized and locally advanced rectal cancer patients (stage II III, without distant metastasis). Based on the major findings, there were no significant differences in most of outcomes between the two interventions. The benefit of the SCRT is more convenient and inexpensive, especially in centers with a long waiting list or lack of medical resources (Bujko et al., 2006; Zhou et al., 2014). Furthermore, LCRT is better than SCRT at increasing pathologic complete response rate. Therefore, based on the available evidence, choose which kind of treatment strategy largely depends on the clinician's experience, the patient's clinical features and the public health resources (Sajid et al., 2010; Rodel et al., 2012; Palta et al., 2014).

Limitations: Only 3 RCTs, total 357 rectal cancer patients, were included in the meta-analysis, so the sample size was too small to detect the possible statistical difference in some outcomes, such as sphincter preservation rate, downstaging rate, local recurrence rate and grade 3 4 acute toxicity, which were with serious or very serious imprecision (Guyatt et al., 2011a). Take grade 3 4 acute toxicity as example, the statistical power of the meta-analysis was only 0.2881 , undoubtedly, it is not enough (Borenstein et al., 2009). In addition, there were some potential risks of biases. Firstly, though all included RCTs mentioned "random", only 1 RCT 
reported the method of adequate randomized sequence generation (Pettersson et al., 2010), 1 RCTs was unclear (Latkauskas et al., 2012), 1 RCT with high risk (Bujko et al., 2013). Secondly, only 1 RCT reported the details about the method of allocation concealment (Pettersson et al., 2010), 1 RCT was unclear (Latkauskas et al., 2012), 1 RCT with high risk (Bujko et al., 2013). So selection biases and attrition biases were inevitable (Higgins and Green, 2011).

It should be noted that clinical heterogeneity existed in individual studies. There were some possible sources of clinical heterogeneity. Firstly, though most recruited rectal cancer patients with clinical stage II $\sim$ III, there was still some stage I and IV patients, and that the proportion of different clinical stage of the patients included in each study was not completely comparable, which might be an important source of clinical heterogeneity. Secondly, the type and dose of the same chemotherapeutics, as well as route of administration in each study, was not comparable, which may introduce heterogeneity. Thirdly, TME was performed in most studies, except the study performed by Bujko et al 2013, in which local excision was adopted (Bujko et al., 2013). Last but not least, duration of follow-up was different in each study. In one word, these limitations might lead to potential biases in the systematic review process. In addition, all included RCTs did not report long-term follow up results, such as overall survival, progression free survival and distant metastases recurrence rate. Therefore we could not objectively and comprehensively evaluation the efficacy of two treatment strategies and further studies were needed to address this issue.

In conclusion, SCRT with delayed surgery is as effective as LCRT with delayed surgery for treatment of rectal cancer in terms of sphincter preservation rate, local recurrence rate, grade 3 4 acute toxicity, R0 resection rate and downstaging rate. LCRT significantly increased pCR rate compared with SCRT. Due to serious risk of bias and imprecision, this conclusion need further multicenter large samples RCTs with longer-term follow up to confirm it.

\section{References}

Balshem H, Helfand M, Schunemann HJ, et al (2011). GRADE guidelines: 3 . Rating the quality of evidence. J Clin Epidemiol, 64, 401-6.

Borenstein M, Hedges LV, Higgins JPT, et al 2009. Introduction to Meta-Analysis, John Wiley \& Sons, Ltd.

Bujko K, Bujko M (2011). Point: short-course radiation therapy is preferable in the neoadjuvant treatment of rectal cancer. Semin Radiat Oncol, 21, 220-7.

Bujko K, Nowacki MP, Nasierowska-Guttmejer A, et al (2006). Long-term results of a randomized trial comparing preoperative short-course radiotherapy with preoperative conventionally fractionated chemoradiation for rectal cancer. Br J Surg, 93, 1215-23.

Bujko K, Partycki M, Pietrzak L (2014). Neoadjuvant radiotherapy ( 5 x 5 Gy): immediate versus delayed surgery. Recent Results Cancer Res, 203, 171-87.

Bujko K, Richter $P$, Smith FM, et al (2013). Preoperative radiotherapy and local excision of rectal cancer with immediate radical re-operation for poor responders: A prospective multicentre study. Radiotherapy Oncol, 106, 198-205.

Frykholm GJ, Glimelius B, Pahlman L (1993). Preoperative or postoperative irradiation in adenocarcinoma of the rectum: final treatment results of a randomized trial and an evaluation of late secondary effects. Dis Colon Rectum, 36, 564-72.

Colorectal Cancer Collaborative Group (2001). Adjuvant radiotherapy for rectal cancer: a systematic overview of 8,507 patients from 22 randomised trials. Lancet, 358, 1291-304.

Guyatt GH, Oxman AD, Kunz R, et al (2011a). GRADE guidelines 6 . Rating the quality of evidence-imprecision. $J$ Clin Epidemiol, 64, 1283-93.

Guyatt GH, Oxman AD, Vist G, et al (2011b). GRADE guidelines: 4. Rating the quality of evidence--study limitations (risk of bias). J Clin Epidemiol, 64, 407-15.

Guyatt GH, Oxman AD, Vist G, et al (2011c). GRADE guidelines: 4. Rating the quality of evidence-study limitations (risk of bias). J Clin Epidemiol, 64, 407-15.

Hatfield $P$, Hingorani M, Radhakrishna G, et al (2009). Shortcourse radiotherapy, with elective delay prior to surgery, in patients with unresectable rectal cancer who have poor performance status or significant co-morbidity. Radiother Oncol, 92, 210-4.

Higgins JPT, Green S (2011). Cochrane Handbook for Systematic Reviews of Interventions Version 5.1.0 [updated March 2011]. Available from www.cochrane-handbook.org.

Kapiteijn E, Marijnen CA, Nagtegaal ID, et al (2001). Preoperative radiotherapy combined with total mesorectal excision for resectable rectal cancer. $N$ Engl J Med, 345, 638-46.

Kye BH, Cho HM (2014). Overview of radiation therapy for treating rectal cancer. Ann Coloproctol, 30, 165-74.

Latkauskas T, Pauzas H, Gineikiene I, et al (2012). Initial results of a randomized controlled trial comparing clinical and pathological downstaging of rectal cancer after preoperative short-course radiotherapy or long-term chemoradiotherapy, both with delayed surgery. Colorectal Dis, 14, 294-8.

Lee WC, Yusof MM, Lau FN, et al (2013). Preoperative long course chemoirradiation in a developing country for rectal carcinoma: Kuala Lumpur hospital experience. Asian Pac J Cancer Prev, 14, 3941-4.

Minsky BD (2011). Counterpoint: long-course chemoradiation is preferable in the neoadjuvant treatment of rectal cancer. Semin Radiat Oncol, 21, 228-33.

Minsky BD, Rodel C, Valentini V (2014). Preoperative therapy for rectal cancer: Short-course radiation vs. long-course chemoradiation. Seminars Colon Rectal Surgery, 25, 19-21.

Nilsson PJ, van Etten B, Hospers GAP, et al (2013). Short-course radiotherapy followed by neo-adjuvant chemotherapy in locally advanced rectal cancer - the RAPIDO trial. $B M C$ Cancer, 13.

Palta M, Willett CG, Czito BG (2014). Short-Course versus Long-Course Chemoradiation in Rectal Cancer-Time to Change Strategies? Curr Treat Options Oncol, [Epub ahead of print].

Pettersson D, Cedermark B, Holm T, et al (2010). Interim analysis of the Stockholm III trial of preoperative radiotherapy regimens for rectal cancer. Br J Surg, 97, 580-7.

Radu C, Berglund A, Pahlman L, et al (2008). Short-course preoperative radiotherapy with delayed surgery in rectal cancer - a retrospective study. Radiother Oncol, 87, 343-9.

Rodel C, Trojan J, Bechstein WO, et al (2012). Neoadjuvant short- or long-term radiochemotherapy for rectal cancer: how and who should be treated? Dig Dis, 30, 102-8.

Sajid MS, Siddiqui MR, Kianifard B, et al (2010). Short-course 
Shi-Xin Liu et al

versus long-course neoadjuvant radiotherapy for lower rectal cancer: a systematic review. Ir J Med Sci, 179, 165-71.

Sauer R, Becker H, Hohenberger W, et al (2004). Preoperative versus postoperative chemoradiotherapy for rectal cancer. $N$ Engl J Med, 351, 1731-40.

Sauer R, Liersch T, Merkel S, et al (2012). Preoperative versus postoperative chemoradiotherapy for locally advanced rectal cancer: results of the German CAO/ARO/AIO-94 randomized phase III trial after a median follow-up of 11 years. J Clin Oncol, 30, 1926-33.

Sebag-Montefiore D, Stephens RJ, Steele R, et al (2009). Preoperative radiotherapy versus selective postoperative chemoradiotherapy in patients with rectal cancer (MRC CR07 and NCIC-CTG C016): a multicentre, randomised trial. Lancet, 373, 811-20.

SWEDISH RECTAL CANCER TRIAL (1997). Improved survival with preoperative radiotherapy in resectable rectal cancer. $N$ Engl J Med, 336, 980-7.

Zhou ZR, Liu SX, Zhang TS, et al (2014). Short-course preoperative radiotherapy with immediate surgery versus long-course chemoradiation with delayed surgery in the treatment of rectal cancer: a systematic review and metaanalysis. Surg Oncol, 23, 211-21. 